\title{
Electroelastic Actuator Nano- and Microdisplacement for Precision Mechanics
}

\author{
Sergey Mikhailovich Afonin \\ Department of Intellectual Technical Systems, National Research University of Electronic Technology (MIET), Moscow, Russia \\ Email address: \\ eduems@mail.ru \\ To cite this article: \\ Sergey Mikhailovich Afonin. Electroelastic Actuator Nano- and Microdisplacement for Precision Mechanics. American Journal of \\ Mechanics and Applications. Vol. 6, No. 1, 2018, pp. 17-22. doi: 10.11648/j.ajma.20180601.14
}

Received: December 28, 2017; Accepted: January 29, 2018; Published: March 6, 2018

\begin{abstract}
In the present work the structural-parametric model of the piezoactuator is determined in contrast electrical equivalent circuit types Cady or Mason for the calculation of the piezoelectric transmitter and receiver, the vibration piezoactuator and the vibration piezomotor with the mechanical parameters in form the velosity and the pressure. The aim of this work is to obtain the structural-parametric model of the electroelastic actuator with the mechanical parameters the displacement and the force. The method of mathematical physics is used. Structural scheme of electroelastic actuator for nanotechnology is obtained. The transfer functions of the actuators are determined. For calculations control systems for nanotechnology with piezoactuator the structural scheme and the transfer functions of piezoactuator are obtained. The generalized structural-parametric model, the generalized structural scheme, the generalized matrix equation for the electroelastic actuator nano- and microdisplacement are obtained in the matrix form. The deformations of the electroelastic actuator for the precision mechanics are described by the matrix equation.
\end{abstract}

Keywords: Electroelastic Actuator, Piezoactuator, Nanodisplacement, Structural Model and Scheme, Transfer Function

\section{Introduction}

The electroelastic actuator based on the electroelasticity in the form the piezoelectric, piezomagnetic, electrostriction effects is used for the precision mechanics in the nanotechnology, the nanobiology, the microelectronics, the astronomy and the adaptive optics. This actuator are solved problems of the compensation of the temperature and gravity deformations, the correction of the wave front and the precision alignment $[1-10]$. Piezoactuator is the piezomechanical device intended for the actuation of the mechanisms, the systems or the management based on the piezoelectric effect, converts the electrical signals into the mechanical movement and the force $[1,6,9]$.

Piezoactuator nano- and microdisplacement for the precision mechanics provide the movement range from several nanometers to tens of microns, the sensitivity of up to $1 \mathrm{~nm} / \mathrm{V}$, the loading capacity of up to $1000 \mathrm{~N}$. Piezoactuator give high stress and speed of operation, return to the initial state when switched off and have very low relative displacement less than $1 \%$. Piezoactuator nano- and microdisplacement is used in the majority of the scanning tunneling microscopes, the scanning force microscopes, the atomic force microscopes [1 - 20].

The structural-parametric model of the piezoactuator is determined in contrast electrical equivalent circuit types Cady or Mason for the calculation of the piezoelectric transmitter and receiver, the vibration piezoactuator and the vibration piezomotor with the mechanical parameters in form the velosity and the pressure $[2-5,11,12]$. By using the method of mathematical physics and solving the wave equation with the Laplace transform for the corresponding equations of the piezoeffect $[6,9,10,20]$, the boundary conditions on loaded faces of the piezoactuator, the strains along the coordinate axes, it is possible to construct the structural parametric model of the piezoactuator $[14,15]$. Its transfer functions and structural scheme are determined.

The generalized structural-parametric model and structural scheme, the generalized matrix equation for the electroelastic actuator nano- and microdisplacement are obtained in the matrix form in general from the wave equation of the actuator and the equation of the electroelasticity. 


\section{Structural Model and Scheme}

For clarity, let us consider the problems of the piezoelasticity. As the result of the joint solution of the wave equation of the piezoactuator nano- and microdisplacement equation with the Laplace transform, the piezoeffect equation and the boundary conditions on the two loaded working surfaces of the piezoactuator, we obtain the corresponding structural-parametric model of the piezoactuator $[15,16]$. The aim of this work is to obtain the structural-parametric model of the electroelastic actuator with the mechanical parameters the displacement and the force.

For piezoactuator the deformation corresponds to stressed state. If stress $T$ is created in piezoactuator, the deformation $S$ is formed in it. There are six stress components $T_{1}, T_{2}$, $T_{3}, T_{4}, T_{5}, T_{6}$, the components $T_{1}-T_{3}$ are related to extension-compression stresses, $T_{4}-T_{6}$ to shear stresses.

The matrix state equations $[7,9,10]$ connecting the electric and elastic variables for piezoceramics have the form

$$
\begin{aligned}
& (\mathbf{D})=(\mathbf{d})(\mathbf{T})+\left(\boldsymbol{\varepsilon}^{\mathrm{T}}\right)(\mathbf{E}) \\
& (\mathbf{S})=\left(\mathbf{s}^{\mathrm{E}}\right)(\mathbf{T})+(\mathbf{d})^{\mathrm{t}}(\mathbf{E})
\end{aligned}
$$

The first equation describes the direct piezoelectric effect, and the second equation records the inverse piezoelectric effect; (S) is the column matrix of relative deformations; (T) is the column matrix of mechanical stresses; (E) is the column matrix of electric field strength along the coordinate axes; (D) is the column matrix of electric induction along the coordinate axes; $\left(\mathbf{s}^{\mathrm{E}}\right)$ is the elastic compliance matrix for $E=$ const ; and $(\mathbf{d})^{t}$ is the transposed matrix of the piezomodules, $\left(\boldsymbol{\varepsilon}^{\mathrm{T}}\right)$ is the matrix of dielectric constants for $T=$ const

For polarized ceramics PZT there are five independent components $s_{11}^{E}, s_{12}^{E}, s_{13}^{E}, s_{33}^{E}, s_{55}^{E}$ in the elastic compliance matrix.

Let us consider the electroelastic actuator.

In general the equation of electroelasticity $[10,12,15]$ has following form

$$
S_{i}=d_{m i} \Psi_{m}(t)+s_{i j}^{\Psi} T_{j}(x, t)
$$

where $S_{i}=\partial \xi(x, t) / \partial x$ is the relative displacement along axis $i$ of the cross section of the piezoactuator or the piezoplate, $\Psi_{m}=\left\{E_{m}, D_{m}\right.$ is the control parameter $E$ for the voltage control, $D$ for the current control along axis $m, T_{j}$ is the mechanical stress along axis $j, d_{m i}$ is the coefficient of electroelasticity, for example, piezomodule, $s_{i j}^{\Psi}$ is the elastic compliance for control parameter $\Psi=$ const .

The piezoactuator for nano- and microdisplacement on Figure 1 has the following properties: $\delta$ is the thickness, $h$ is the height, $b$ is the width, respectively $l=\{\delta, h, b$ the length of the piezoactuator for the longitudinal, transverse and shift piezoeffect. The direction of the polarization axis $P$, i.e. the direction along which polarization was performed, is usually taken as the direction of axis 3 .

The equation of the inverse piezoeffect for controlling voltage has the form

$$
S_{i}=\partial \xi(x, t) / \partial x, \Psi_{m}(t)=E_{m}(t)=U(t) / \delta
$$

where $S_{i}$ is the relative displacement of the cross section of the piezoactuator along axis $i, \xi(x, t)$ is the displacement of the section of the piezoactuator, $d_{m i}$ is the piezomodule, $E_{m}(t)$ is the electric field strength along axis $m, U(t)$ is the voltage between the electrodes, $s_{i j}^{E}$ is the elastic compliance for $E=$ const , indexes $i, j=1,2, \ldots, 6 ; m=1,2,3$. The main size or the working length $l=\{\delta, h, b$ for the piezoactuator, respectively, the thickness, the height and the width for the longitudinal, transverse and shift piezoeffect.

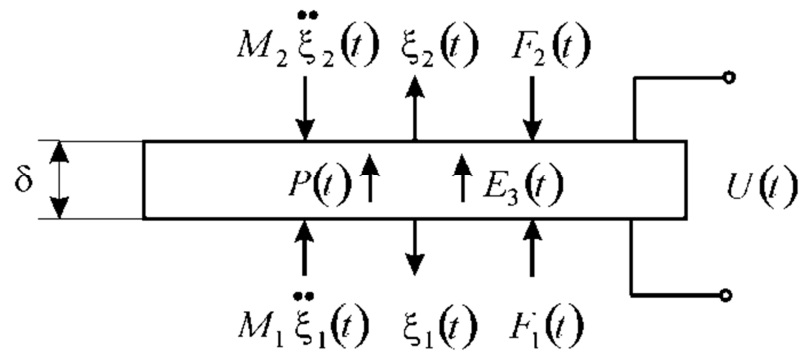

a)

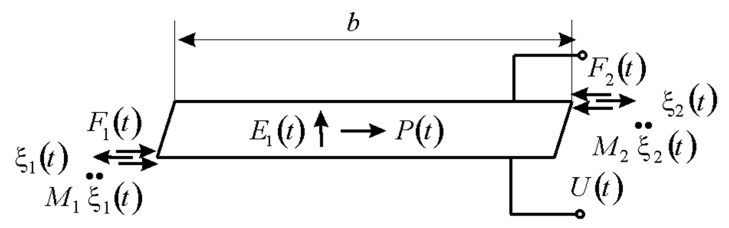

b)

Figure 1. Piezoactuator a) for the longitudinal piezoeffect, b) for the shift piezoeffect.

For calculation of the electroelastic actuator nano- and microdisplacement is used the wave equation $[10,12,16,19]$ for the wave propagation in a long line with damping but without distortions. After Laplace transform is obtained the linear ordinary second-order differential equation with the parameter $p$, where the original problem for the partial differential equation of hyperbolic type using the Laplace transform is reduced to the simpler problem $[10,13,14]$ for the linear ordinary differential equation

$$
\frac{d^{2} \Xi(x, p)}{d x^{2}}-\gamma^{2} \Xi(x, p)=0
$$

with its solution

$$
\Xi(x, p)=C e^{-x \gamma}+B e^{x \gamma}
$$


where $\Xi(x, p)$ is the Laplace transform of the displacement of the section of the electroelastic actuator, $\gamma=p / c^{\Psi}+\alpha$ is the propagation coefficient, $c^{\Psi}$ is the sound speed for $\Psi=$ const,$\alpha$ is the damping coefficient.

The constants $C$ and $B$ of the solution the linear ordinary second-order differential equation [7] are determined from the boundary conditions for the electroelastic actuator

$$
\begin{aligned}
& \Xi(0, p)=\Xi_{1}(p) \text { for } x=0 \\
& \Xi(l, p)=\Xi_{2}(p) \text { for } x=l
\end{aligned}
$$

whence we obtain

$$
C=\frac{\Xi_{1} e^{l \gamma}-\Xi_{2}}{2 \operatorname{sh}(l \gamma)}, B=\frac{\Xi_{2}-\Xi_{1} e^{-l \gamma}}{2 \operatorname{sh}(l \gamma)}
$$

Therefore, the solution the linear ordinary second-order differential equation (5) can be written in the form

$$
\Xi(x, p)=\frac{\Xi_{1}(p) \operatorname{sh}[(l-x) \gamma]+\Xi_{2}(p) \operatorname{sh}(x \gamma)}{\operatorname{sh}(l \gamma)}
$$

The system of the equations for the forces on the faces of the electroelastic actuator are determined in the following form

$$
\begin{aligned}
& \Xi_{1}(p)=\left[1 /\left(M_{1} p^{2}\right)\right] . \\
& \cdot\left\{-F_{1}(p)+\left(1 / \chi_{i j}^{\Psi}\right)\left[v_{m i} \Psi_{m}(p)-[\gamma / \operatorname{sh}(l \gamma)]\left[\operatorname{ch}(l \gamma) \Xi_{1}(p)-\Xi_{2}(p)\right]\right]\right\} \\
& \Xi_{2}(p)=\left[1 /\left(M_{2} p^{2}\right)\right] . \\
& \cdot\left\{-F_{2}(p)+\left(1 / \chi_{i j}^{\Psi}\right)\left[v_{m i} \Psi_{m}(p)-[\gamma / \operatorname{sh}(l \gamma)]\left[\operatorname{ch}(l \gamma) \Xi_{2}(p)-\Xi_{1}(p)\right]\right]\right\}
\end{aligned}
$$

$$
\left.T_{j}(l, p)\right) S_{0}=-F_{2}(p)-M_{2} p^{2} \Xi_{1}(p) \text { for } x=l,
$$

where $T_{j}(0, p)$ and $T_{j}(l, p)$ are the mechanical stress.

From equations of forces acting on the faces of the electroelastic actuator nano- and microdisplacement we obtain the boundary conditions on loaded surfaces

$$
T_{j}(0, p)=\left.\frac{1}{s_{i j}^{\Psi}} \frac{d \Xi(x, p)}{d x}\right|_{x=0}-\frac{d_{m i}}{s_{i j}^{\Psi}} \Psi_{m}(p)
$$

where $S_{0}$ is the cross section area and $M_{1}, M_{2}$ are the displaced mass on the faces of the electroelastic actuator.

From (4), the boundary conditions on loaded surfaces (5), the strains along the axes the system of equations for the generalized structural-parametric model and the generalized parametric structural scheme are determined for Figure 1 of the electroelastic actuator with the output parameters the Laplace transform for the displacements $\Xi_{1}(p), \Xi_{2}(p)$ for the faces of the electroelastic actuator nano- and microdisplacement for the precision mechanics in the form

where $\chi_{i j}^{\Psi}=\frac{s_{i j}^{\Psi}}{S_{0}}, v_{m i}=\left\{\begin{array}{l}d_{33}, d_{31}, d_{15} \\ g_{33}, g_{31}, g_{15}\end{array}, \Psi_{m}=\left\{\begin{array}{l}E_{3}, E_{3}, E_{1} \\ D_{3}, D_{3}, D_{1}\end{array}\right.\right.$,

$s_{i j}^{\Psi}=\left\{\begin{array}{l}s_{33}^{E}, s_{11}^{E}, s_{55}^{E} \\ s_{33}^{D}, s_{11}^{D}, s_{55}^{D}\end{array} \quad, \quad l=\left\{\delta, h, b \quad, \quad c^{\Psi}=\left\{c^{E}, c^{D} \quad\right.\right.\right.$,

$\gamma^{\Psi}=\left\{\gamma^{E}, \gamma^{D}\right.$,

$v_{m i}$ is the coefficient of the electroelasticity, for example, piezomodule, $g_{m i}$ is the piezomodule for the currentcontrolled piezoactuator, $F_{1}(p), F_{2}(p)$ are the Laplace transform of the forces on the faces. Figure 2 shows the generalized structural scheme of the electroelastic actuator nano- and microdisplacement corresponding to the set of equations (10) for the Laplace transform of the displacements of the faces.

The generalized transfer functions of the of the electroelastic actuator are the ratio of the Laplace transform of the displacement of the face actuator and the Laplace transform of the corresponding control parameter or the force at zero initial conditions.

The generalized structural scheme and the generalized transfer functions of the electromagnetoelastic actuator nanoand microdisplacement are obtained from the generalized structural parametric model of the electromagnetoelastic actuator for the precision mechanics. 


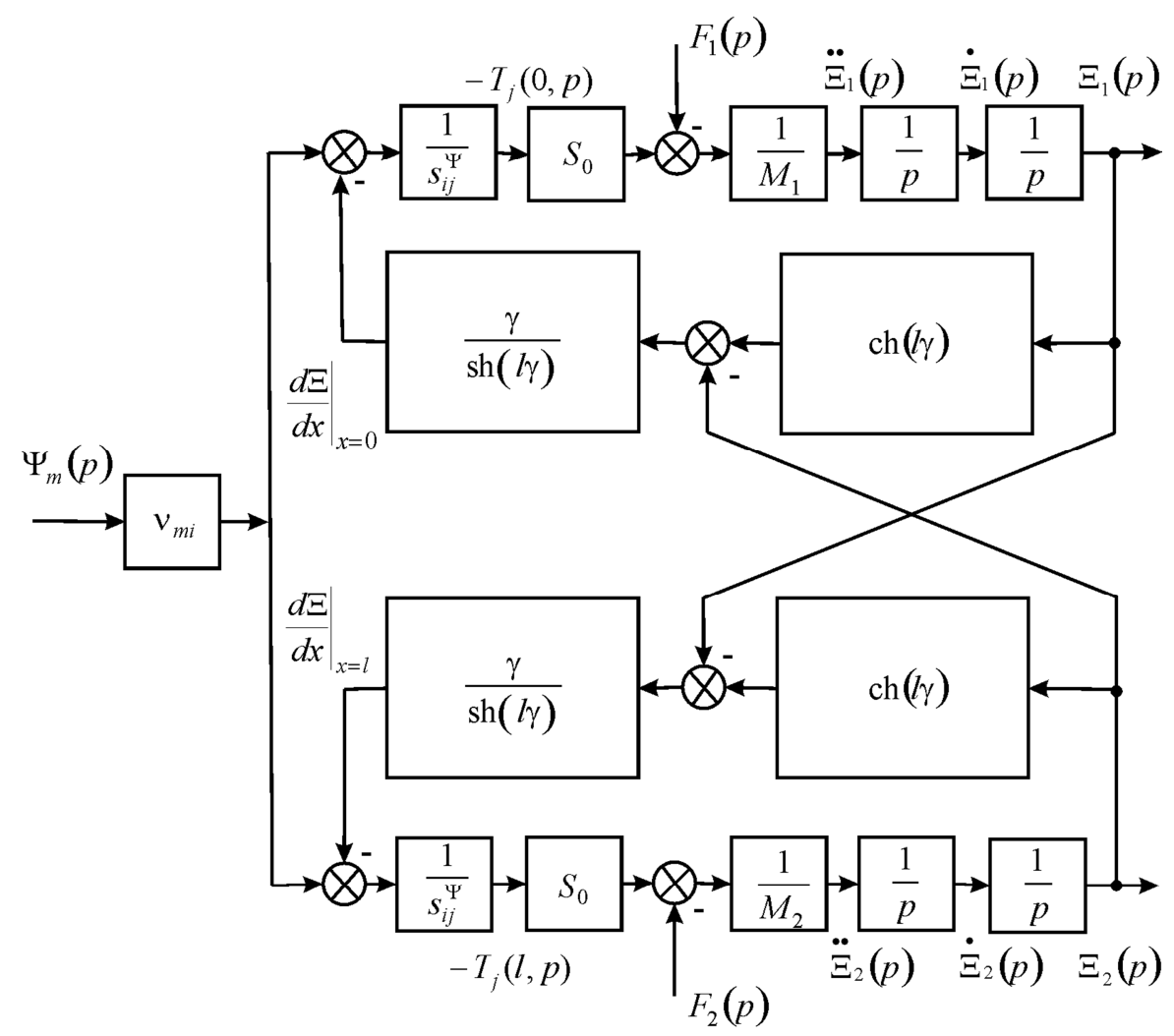

Figure 2. Generalized structural scheme of electroelastic actuator.

\section{Transfer Functions}

The transfer functions of the electroelastic actuator nanoand microdisplacement are determined from its generalized structural-parametric model, taking into account the generalized equation of electroelasticity, its wave equation and the equation of the forces on its faces.

Therefore, the Laplace transforms of displacements for two faces of the actuator are dependent from the Laplace transforms of the general parameter of control and forces on two faces and are written in the matrix form. From (10) for the Laplace transforms of the displacements of two faces of the actuator yields the matrix equation in the following form

$$
\left(\begin{array}{c}
\Xi_{1}(p) \\
\Xi_{2}(p)
\end{array}\right)=\left(\begin{array}{lll}
W_{11}(p) & W_{12}(p) & W_{13}(p) \\
W_{21}(p) & W_{22}(p) & W_{23}(p)
\end{array}\right)\left(\begin{array}{c}
\Psi_{m}(p) \\
F_{1}(p) \\
F_{2}(p)
\end{array}\right)
$$

where the transfer functions

$$
\begin{aligned}
& W_{11}(p)=\frac{\Xi_{1}(p)}{\Psi_{m}(p)}=\frac{v_{m i}}{A_{i j}}\left[M_{2} \chi_{i j}^{\Psi} p^{2}+\gamma \operatorname{th}\left(\frac{l \gamma}{2}\right)\right] \\
& A_{i j}=M_{1} M_{2}\left(\chi_{i j}^{\Psi}\right)^{2} p^{4}+\frac{\left(M_{1}+M_{2}\right) \chi_{i j}^{\Psi}}{c \operatorname{th}(l \gamma)} p^{3}+ \\
& +\left[\frac{\left(M_{1}+M_{2}\right) \chi_{i j}^{\Psi} \alpha}{\operatorname{th}(l \gamma)}+\frac{1}{c^{2}}\right] p^{2}+\frac{2 \alpha}{c} p+\alpha^{2}
\end{aligned}
$$

$$
\begin{gathered}
W_{21}(p)=\frac{\Xi_{2}(p)}{\Psi_{m}(p)}=\frac{v_{m i}}{A_{i j}}\left[M_{1} \chi_{i j}^{\Psi} p^{2}+\gamma \operatorname{th}\left(\frac{l \gamma}{2}\right)\right] \\
W_{12}(p)=\frac{\Xi_{1}(p)}{F_{1}(p)}=-\frac{\chi_{i j}^{\Psi}}{A_{i j}}\left[M_{2} \chi_{i j}^{\Psi} p^{2}+\frac{\gamma}{\operatorname{th}(l \gamma)}\right] \\
W_{13}(p)=\frac{\Xi_{1}(p)}{F_{2}(p)}=W_{22}(p)=\frac{\xi_{2}(p)}{F_{1}(p)}=\frac{\chi_{i j}^{\Psi} \gamma}{A_{i j} \operatorname{sh}(l \gamma)} \\
W_{23}(p)=\frac{\Xi_{2}(p)}{F_{2}(p)}=-\frac{\chi_{i j}^{\Psi}}{A_{i j}}\left[M_{1} \chi_{i j}^{\Psi} p^{2}+\frac{\gamma}{\operatorname{th}(l \gamma)}\right]
\end{gathered}
$$

Let us find the displacement of the faces the electroelastic actuator in the stationary regime for $\Psi_{m}(t)=\Psi_{m 0} \cdot 1(t)$, $F_{1}(t)=F_{2}(t)=0$ and inertial load.

The static displacement of the faces the electroelastic actuator $\xi_{1}(\infty)$ and $\xi_{2}(\infty)$ can be written in the following form

$$
\begin{array}{r}
\xi_{1}(\infty)=\lim _{t \rightarrow \infty} \xi_{1}(t)=\frac{v_{m i} l \Psi_{m 0}\left(M_{2}+m / 2\right)}{M_{1}+M_{2}+m} \\
\xi_{2}(\infty)=\lim _{t \rightarrow \infty} \xi_{2}(t)=\frac{v_{m i} l \Psi_{m 0}\left(M_{1}+m / 2\right)}{M_{1}+M_{2}+m} \\
\xi_{1}(\infty)+\xi_{2}(\infty)=\lim _{t \rightarrow \infty}\left(\xi_{1}(t)+\xi_{2}(t)\right)=v_{m i} l \Psi_{m 0}
\end{array}
$$


where $m$ is the mass of the electroelastic actuator, $M_{1}, M_{2}$ are the load masses.

Let us consider a numerical example for $d_{33}=4 \cdot 10^{-10}$ $\mathrm{m} / \mathrm{V}, U=200 \mathrm{~V}, M_{1}=1 \mathrm{~kg}$ and $M_{2}=4 \mathrm{~kg}$ we obtain the static displacements of the faces of the piezoactuator $\xi_{1}(\infty)=64 \mathrm{~nm}, \xi_{2}(\infty)=16 \mathrm{~nm}, \xi_{1}(\infty)+\xi_{2}(\infty)=80 \mathrm{~nm}$.

After transformation we obtain the expression the transfer function for the voltage-controlled piezoactuator under the longitudinal piezoeffect at zero source resistance with one face rigidly fixed in the following form

$$
W_{21}(p)=\frac{\Xi_{2}(p)}{E_{3}(p)}=\frac{d_{33} \delta}{M_{2} \delta \chi_{33}^{E} p^{2}+\delta \gamma \operatorname{cth}(\delta \gamma)}
$$

From (15) using the approximation of the hyperbolic cotangent by two terms of the power series at $m<<M_{2}$ and $0<\omega<0,01 c^{E} / \delta$ we obtain transfer function

$$
\begin{gathered}
W_{21}(p)=\frac{\Xi_{2}(p)}{E_{3}(p)}=\frac{d_{33} \delta}{T_{t}^{2} p^{2}+2 T_{t} \xi_{t} p+1} \\
T_{t}=\sqrt{M_{2} / C_{33}^{E}}, \xi_{t}=(\alpha \delta / 3) \sqrt{m / M_{2}}, C_{33}^{E}=S_{0} /\left(s_{33}^{E} \delta\right)
\end{gathered}
$$

where $T_{t}$ is the time constant and $\xi_{t}$ is the damping coefficient, $C_{33}^{E}$ is the is rigidity of the piezoactuator.

For the approximation of the hyperbolic cotangent by two terms of the power series, the following expressions of the transfer function of the voltage-controlled piezoactuator at zero source resistance is obtained for the elastic-inertial load at $M_{1} \rightarrow \infty, m<<M_{2}$ under the transverse piezoeffect

$$
\begin{gathered}
W(p)=\frac{\Xi_{2}(p)}{U(p)}=\frac{d_{31} h / \delta}{\left(1+C_{e} / C_{11}^{E}\right)\left(T_{t}^{2} p^{2}+2 T_{t} \xi_{t} p+1\right)} \\
T_{t}=\sqrt{M_{2} /\left(C_{e}+C_{11}^{E}\right)}, \xi_{t}=\alpha h^{2} C_{11}^{E} /\left(3 c^{E} \sqrt{M_{2}\left(C_{e}+C_{11}^{E}\right)}\right)
\end{gathered}
$$

where $U(p)$ is the Laplace transform of the voltage, $T_{t}$ is the time constant and $\xi_{t}$ is the damping coefficient of the piezoactuator.

Therefore, we obtain on Figure 3 for (17) the structural scheme of the voltage-controlled piezoactuator at zero source resistance with one fixed face under the transverse piezoeffect for the elastic-inertial load.

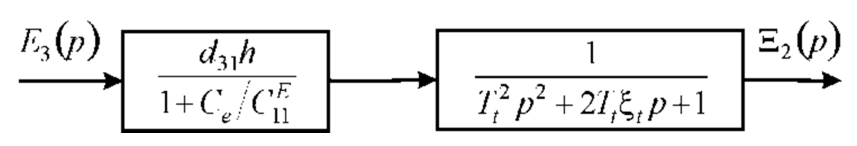

Figure 3. Structural scheme of voltage-controlled piezoactuator at zero source resistance with one fixed face for elastic-inertial load.

The expression for the transient response of the voltagecontrolled piezoactuator under the transverse piezoeffect for the elastic-inertial load is determined

$$
\begin{gathered}
\xi(t)=\xi_{m}\left[1-\frac{e^{-\frac{\xi_{t} t}{T_{t}}}}{\sqrt{1-\xi_{t}^{2}}} \sin \left(\omega_{t} t+\phi_{t}\right)\right] \\
\xi_{m}=\frac{d_{31}(h / \delta) U_{m}}{1+C_{e} / C_{11}^{E}}, \omega_{t}=\frac{\sqrt{1-\xi_{t}^{2}}}{T_{t}}, \phi_{t}=\operatorname{arctg}\left(\frac{\sqrt{1-\xi_{t}^{2}}}{\xi_{t}}\right)
\end{gathered}
$$

where $\xi_{m}$ is the steady-state value of displacement for the voltage-controlled piezoactuator, $U_{m}$ is the amplitude of the voltage in the steady-state.

Let us consider a numerical example for the voltagecontrolled piezoactuator from the piezoceramics PZT under the transverse piezoelectric effect with one fixed face for the elastic-inertial load $M_{1} \rightarrow \infty, m<<M_{2}$ and input voltage with amplitude $U_{m}=50 \mathrm{~V}$ at $d_{31}=2.5 \cdot 10^{-10} \mathrm{~m} / \mathrm{V}, h / \delta=20$, $M_{2}=4 \mathrm{~kg}, C_{11}^{E}=2 \cdot 10^{7} \mathrm{~N} / \mathrm{m}, C_{e}=0.5 \cdot 10^{7} \mathrm{H} / \mathrm{m}$ we obtain values the steady-state value of displacement and the time constant $\xi_{m}=200 \mathrm{~nm}, T_{t}=0.4 \cdot 10^{-3} \mathrm{c}$.

Therefore, for calculations control systems with the piezoactuator for the precision mechanics the structural scheme and the transfer functions of the piezoactuator nanoand microdisplacement for the precision mechanics are obtained.

\section{Results and Discussions}

We obtain the structural scheme of the electroelastic actuator nano- and microdisplacement for the precision mechanics. From generalized structural-parametric model of the electroelastic actuator after algebraic transformations we obtain the transfer functions of the electroelastic actuator.

It is possible to construct the generalized structuralparametric model using the solutions of the wave equation of the actuator and taking into account the features of its deformations along the coordinate axes.

For calculations control systems in the nanotechnology, the nanobiology, the microelectronics, the astronomy and the adaptive optics with the electroelastic actuator nano- and microdisplacement for the precision mechanics its transfer functions are obtained.

\section{Conclusions}

Taking into account the features of the deformations along the axes and using the solutions of the wave equation, it is possible to construct the structural-parametric model and structural scheme of the electroelastic actuator nano- and microdisplacement for the precision mechanics and to describe its dynamic and static properties.

The structural scheme and the transfer functions of the piezoactuator are obtained from structural parametric model 
of the piezoactuator for the precision mechanics.

The generalized structural-parametric model, the generalized structural scheme, the generalized matrix equaion for the electroelastic actuator nano- and microdisplacement in the matrix form with the output parameters displacements are obtained.

The structural-parametric models, the structural schemes of the piezoactuator for the transverse, longitudinal, shift piezoelectric effects are determined from the generalized structural-parametric model of the electroelastic actuator nano- and microdisplacement for the precision mechanics.

From the solution of the wave equation, the equations of the electroelasticity and the deformations along the axes with using the Laplace transform, the generalized structuralparametric model and the generalized structural scheme of the electroelastic actuator nano- and microdisplacement with the mechanical parameters the displacement and the force are constructed for the precision mechanics.

The deformations of the electroelastic actuator for the precision mechanics are described by the matrix equation for the transfer functions of the actuator.

\section{References}

[1] J. Schultz, J. Ueda, H. Asada, Cellular actuators. Oxford: Butterworth-Heinemann Publisher. 2017, 382 p.

[2] S. Zhou, Z Yao, "Design and optimization of a modalindependent linear ultrasonic motor," IEEE transaction on ultrasonics, ferroelectrics, and frequency control 61, 3, 535546 (2014), doi:10.1109/TUFFC.2014.2937.

[3] J. Przybylski, "Static and dynamic analysis of a flextensional transducer with an axial piezoelectric actuation," Engineering structures $84,140-151$ (2015),

doi:10.1016/j.engstruct.2014.11.025.

[4] J. Ueda, T. Secord, H. H. Asada, "Large effective-strain piezoelectric actuators using nested cellular architecture with exponential strain amplification mechanisms," IEEE/ASME transactions on mechatronics 15, 5, 770-782 (2010), doi:10.1109/TMECH.2009.2034973.

[5] M. Karpelson, G.-Y. Wei, R. J. Wood, "Driving high voltage piezoelectric actuators in microrobotic applications," Sensors and actuators A: Physical 176, 78-89 (2012), doi:10.1016/j.sna.2011.11.035.

[6] S. M. Afonin, "Block diagrams of a multilayer piezoelectric motor for nano- and microdisplacements based on the transverse piezoeffect," Journal of computer and systems sciences international 54, 3, 424-439 (2015), doi: $10.1134 / \mathrm{S} 1064230715020021$.

[7] S. M. Afonin, "Absolute stability conditions for a system controlling the deformation of an elecromagnetoelastic transduser," Doklady mathematics 74, 3, 943-948 (2006), doi:10.1134/S1064562406060391.

[8] S. M. Afonin, "Stability of strain control systems of nano-and microdisplacement piezotransducers," Mechanics of solids 49, 2, 196-207 (2014), doi:10.3103/S0025654414020095.

[9] S. M. Afonin, "Structural parametric model of a piezoelectric nanodisplacement transduser," Doklady physics, 53, 3, 137143 (2008), doi:10.1134/S1028335808030063.

[10] S. M. Afonin, "Solution of the wave equation for the control of an elecromagnetoelastic transduser," Doklady mathematics 73, 2, 307-313 (2006), doi:10.1134/S1064562406020402.

[11] W. G. Cady, Piezoelectricity: An introduction to the theory and applications of electromechancial phenomena in crystals. New York, London: McGraw-Hill Book Company. 1946, 806 p.

[12] Physical acoustics: Principles and methods. Vol.1. Part A. Methods and devices. Ed.: W. Mason. New York: Academic Press. 1964. 515 p.

[13] D. Zwillinger, Handbook of differential equations. Boston: Academic Press. 1989. 673 p.

[14] S. M. Afonin, "Structural-parametric model and transfer functions of electroelastic actuator for nano- and microdisplacement," Chapter 9 in Piezoelectrics and nanomaterials: Fundamentals, developments and applications. Ed. I. A. Parinov. New York: Nova Science. 2015, pp. 225-242.

[15] S. M. Afonin, "A structural-parametric model of electroelastic actuator for nano- and microdisplacement of mechatronic system," Chapter 8 in Advances in nanotechnology. Volume 19. Eds. Z. Bartul, J. Trenor. New York: Nova Science. 2017, pp. 259-284.

[16] S. M. Afonin, "Generalized parametric structural model of a compound elecromagnetoelastic transduser," Doklady physics 50, 2, 77-82 (2005), doi:10.1134/1.1881716.

[17] S. M. Afonin, "Generalized hysteresis characteristic of a piezoelectric transducer and its harmonic linearization," Mechanics of solids 39, 6, 14-19 (2004).

[18] S. M. Afonin, "Nano- and micro-scale piezomotors," Russian engineering research 32, 7-8, 519-522 (2012), doi:10.3103/S1068798X12060032.

[19] S. M. Afonin, "Elastic compliances and mechanical and adjusting characteristics of composite piezoelectric transducers," Mechanics of solids 42, 1, 43-49 (2007), doi:10.3103/S0025654407010062.

[20] S. M. Afonin, "Structural-parametric model electromagnetoelastic actuator nanodisplacement for mechatronics," International journal of physics 5, 1, 9-15 (2017). 